\title{
Effects of an Improved Auditory-Periphery Model on the Response Properties of Modeled Neurons in the Dorsal Cochlear Nucleus
}

\author{
Stephen Vetsis, Xiaohan Zheng, Herbert F. Voigt and Ian C. Bruce, Senior Member
}

\begin{abstract}
Dorsal Cochlear Nucleus (DCN) neurons were simulated using two different models of auditory nerve (AN) fibers as inputs, the Carney model [1] and the Zilany \& Bruce model [2], [3]. The change of AN models produced very different responses. DCN neurons simulated using the Zilany and Bruce model had much greater regions of excitation and significantly reduced regions of inhibition. Further investigation into the differences between the two $A N$ models revealed that the broadened tuning of the auditory nerve fibers produced by the Zilany \& Bruce model at higher stimulus intensities coupled with the lower excitation thresholds accounted for the discrepancies. Changes in DCN model connection parameters and their effects on the resulting simulated neurons were then explored in an attempt to provide a qualitative framework for determining a set of DCN parameters capable of reproducing physiological responses using the Zilany and Bruce AN fiber model. A mix of increased W-cell inhibition to narrow the response of type-II cells and changes in AN connection parameters to increase their excitability at higher intensities were found to be required to reproduce physiological response properties. These changes in the type-II cell response would provide adequate compensation for the increased excitation and broadened responses at higher intensities observed in DCN model P-cells.
\end{abstract}

\section{INTRODUCTION}

In a recent study, Zheng \& Voigt presented a computational model of the dorsal cochlear nucleus (DCN) [4]. This study used the 1993 Carney model of the auditory periphery to generate input auditory nerve (AN) fiber spike trains to determine a set of DCN model parameters able to recreate the typical response behaviors of the various cell types in the mammalian (gerbil, cat) DCN [1]. The 1993 Carney model has since received further refinements and additions to allow for the recreation of a wider array of physiological phenomena. The current form of the model has been described by Zilany \& Bruce in two recent papers [2], [3]. Most importantly, this model is able to accurately reproduce the broadened frequency tuning of AN fibers at higher stimulus intensities. Given the complex response properties of DCN neurons to both tonal and broadband stimuli, this physiological phenomenon is expected to have significant impact on the DCN model responses.

The goal of this study is to develop a framework for finding a set of DCN model parameters that can be used to reproduce the response properties of neurons in the

This research was supported by the CIHR NET Grant 54023.

S. Vetsis (vetsissegrads.ece.mcmaster.ca) and I. C. Bruce (ibruce@ieee. org) are with the Dept. of Electrical \& Computer Engineering, McMaster University, Hamilton, ON, L8S 4K1, Canada.

X. Zheng (xhzhenglbu.edu) and H. F. Voigt (hfv@bu.edu) are with the Dept. of Biomedical Engineering \& Hearing Research Center, Boston University, 44 Cummington Street, Boston, MA, 02215-2407, USA. mammalian DCN using the more physiologically accurate Zilany \& Bruce model of the auditory periphery as input. We first explore the changes arising in the response properties of the model DCN neurons and attempt to determine the underlying reasons for these changes. We then examine the effects of DCN model parameter changes and attempt to qualitatively describe a strategy for determining an optimal set of parameters that is able to reproduce physiological responses.

\section{METHODS}

\section{A. The DCN model}

The DCN model used in this study was developed by Zheng and Voigt [4]. The model consists of a network of five different cell groups. The connections within a single frequency slice between the different cell types can be seen in the schematic shown in Fig. 1. AN fibers represent auditory nerve axons. P-cells represent principal cells. By varying the connection parameters in the $\mathrm{DCN}$ circuit, $\mathrm{P}$ cells can produce type III, type IV and type IV-T cell responses. I2-cells represent cells with a type II response maps (RM). W-cells represent wide band inhibitors which receive inputs from a large number of $\mathrm{AN}$ fiber inputs over a large bandwidth. Each cell type receives a different number of AN fiber inputs over different bandwidths of AN fiber characteristic frequencies (CFs).

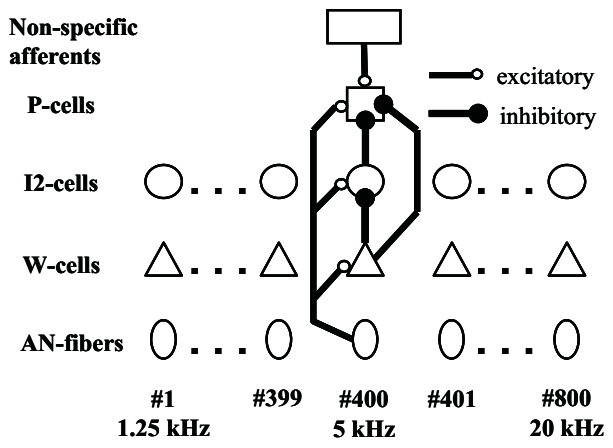

Fig. 1. This schematic represents the organization of the DCN. Reprinted with permission from [4] (c) Springer-Verlag 2006.

The MacGregor neuromine [5] provides the basis for the individual neuron model. The model consists of several parallel pathways with variable conductances and a membrane capacitance. Variable conductances are modeled as step increases at the onset of an input action potential followed by an exponential decay. 


\section{B. Auditory nerve models}

This study uses two different models for AN fibers. The first model is Carney's 1993 model for auditory-nerve fibers in cats [1]. This model includes a time-varying narrow-band filter that represents the mechanical tuning of the basilar membrane (BM). The BM filter's gain and bandwidth is varied over time via a feedback mechanism that produces a compressive nonlinearity similar to that seen in physiological responses. The BM filter is followed by model sections describing the transduction of $\mathrm{BM}$ vibrations to inner hair cell receptor potential, adaptation in the resulting synaptic release, and Poisson-like spike generation with absolute and relative refractoriness.

The second model used in this study was the cat auditory nerve model developed by Zilany \& Bruce [2], [3]. The model, illustrated in Fig. 2, is based on the Carney model and has several improvements to increase its physiological accuracy. The main changes of interest to this study are: i) more accurate shapes of BM threshold tuning curves, ii) improved modeling of the effects of high stimulus levels on $\mathrm{BM}$ tuning, and iii) addition of a middle ear filter.

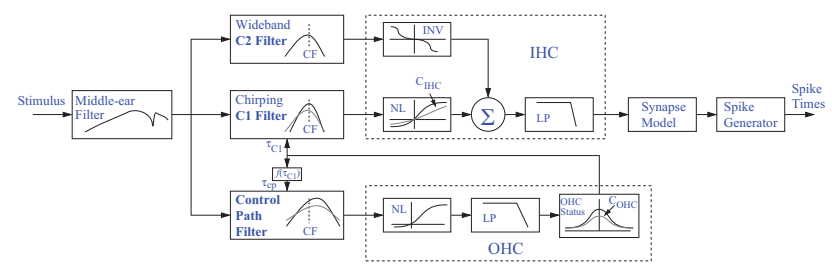

Fig. 2. Zilany \& Bruce cat auditory nerve model. Reprinted with permission from [2], (c) Acoustical Society of America.

For both models, the output is the spike timing information for an AN fiber with a specific preferred or characteristic frequency $(\mathrm{CF})$. In the Zilany \& Bruce model, the parameters $C_{\mathrm{IHC}}$ and $C_{\mathrm{OHC}}$, which control the level of inner and outer hair cell impairment, respectively, can be adjusted to provide a desired hearing threshold shift at a specific CF [2]. For the purposes of this study both parameters were left at values of 1 , indicating normal inner and outer hair cell function.

\section{Simulation protocol}

800 AN fibers with sequential CFs, separated by 0.005 octaves and centered around the DCN model best frequency of $5 \mathrm{kHz}$ were simulated. AN fiber spontaneous rates, with corresponding changes in rate-level functions, were assigned randomly using a physiological distribution [6].

Stimuli were $200 \mathrm{~ms}$ pure tone waveforms, presented once per second with a $10 \mathrm{~ms}$ onset delay and $5 \mathrm{~ms}$ sinusoidal ramp length at each end of the stimulus waveform. Stimulus frequencies were varied by 0.1 octave steps within 1.5 octaves above and below $5 \mathrm{kHz}$. Stimulus intensities were varied from 0 to $90 \mathrm{~dB}$ SPL in $2 \mathrm{~dB}$ SPL steps.

Response maps are displayed as two-dimensional matrices of frequency-level combinations where blue, gray and red regions represent excitatory, spontaneous activity and inhibitory regions, respectively. Spontaneous rates were computed over the last $160 \mathrm{~ms}$ of the dead time following each stimulus. Excitation rates were computed over the last $160 \mathrm{~ms}$ of each applied pure tone waveform. Regions were designated as inhibitory, excitatory or spontaneous according to statistical criteria [7].

Discharge rate plots for the AN fiber responses were plotted as matrices similar to the response maps. Instead of using a statistical comparison to the spontaneous activity, raw discharge rates were plotted as contour maps.

DCN parameters were initially set to the values shown in Figure 4 of Zheng \& Voigt [4]. Parameters were then modified manually and response maps plotted to view effects.

\section{RESULTS}

\section{A. Comparison of the response maps of simulated DCN units using the two different AN fiber models}

Figure 4 of Zheng \& Voigt outlined a set of parameter settings for their DCN model that were able to reproduce the canonical response properties of type III, type IV and type IV-t cells of the DCN. Using the same parameter values, we simulated DCN P-cells using AN fiber inputs produced from both the Carney and Zilany \& Bruce models. The results can be seen in Fig. 3 .

It is evident that the responses produced by using each of the AN models as input differ greatly. The responses obtained using the Zilany \& Bruce model show a much greater level of excitation. The response maps tend to not only produce much broader regions of excitation but tend to be more excitable at significantly lower stimulus intensities. We also notice substantially smaller regions of inhibition, especially at higher stimulus levels in our type IV and type IV-t cells. The type-III units simulated using the Zilany \& Bruce model completely lack a low-frequency region of inhibition.

B. Comparison of the response properties of the AN fiber inputs produced by each AN model

The results shown in Fig. 3 make it extremely clear that the change in AN model inputs significantly alters the response properties of DCN P-cells. Figure 4 compares the response properties of AN fibers with low, medium and high CFs. Carney model fibers can be seen on the left and Zilany and Bruce fibers can be seen on the right.

Figure 4 shows significant differences between the two models. Common across all AN fibers we see a significantly wider tuning at higher intensities for all fibers produced using the Zilany \& Bruce model. We also see much lower excitation thresholds in Zilany \& Bruce model fibers.

The most striking difference between the two models is the presence of the distortions in the Carney model. We see significant levels of excitation at higher frequencies for the low-CF fiber and vice versa for the high-CF fiber.

\section{Effects of DCN connection parameter changes on DCN cell response properties}

While statistically based response maps are excellent tools for examining the response properties of excitable cells, we 


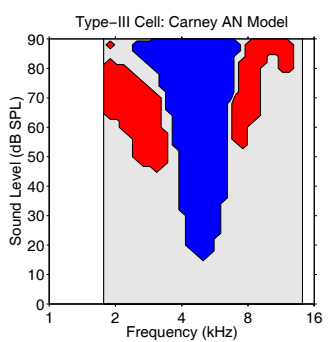

(a)

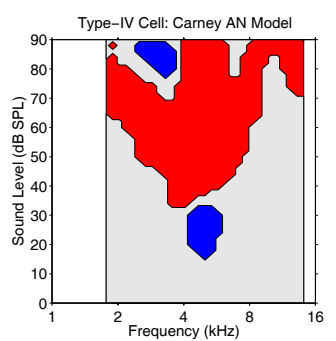

(c)

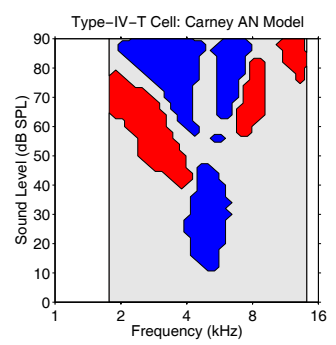

(e)

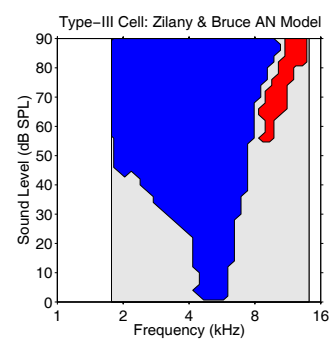

(b)

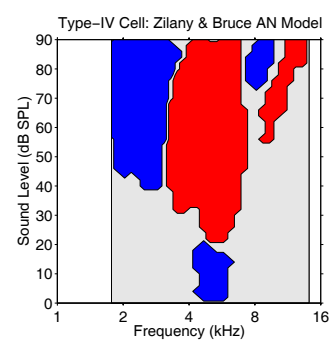

(d)

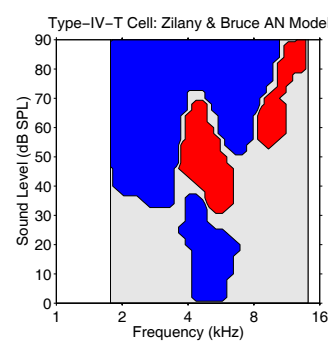

(f)
Fig. 3. Response maps of typical P-cell types are shown. Type III, type IV and type IV-T cells simulated using the Carney AN model are shown in subfigures (a), (c) and (e), respectively. Type III, type IV and type IV-T cells simulated using the Zilany \& Bruce AN model are shown in subfigures (b), (d) and (f), respectively.

will use plots of excitation rates to examine the effects of parameter changes on the response properties of DCN cells. These types of plots provide a more detailed description of the behavior of excitable cells and provide better direction in determining a parameter change strategy.

Figure 5 shows contour plots of the discharge rates of type-II DCN cells using both the Carney and Zilany and Bruce AN fiber models as input. We can see that the type-II cells simulated using the Zilany \& Bruce AN fiber model have much lower thresholds of excitation and much broader regions of excitation at higher frequencies. This is quite similar to the observations of the differences between the two AN fiber response properties. We also notice that the Zilany \& Bruce type-II cells show slightly less excitation at higher frequencies. This observation accounts the for the observed higher levels of excitation at higher frequencies in P-cells simulated using Zilany \& Bruce model AN fibers. Type-II cells provide significant inhibitory input to P-cells and if their responses are weaker at higher stimulus intensities, they will provide less inhibition to the P-cells at those intensities.

Figure 6 shows the W-cell responses arising from each of the AN models. We can see that the $\mathrm{W}$-cell produced using the Carney model has an extra region of excitation in the

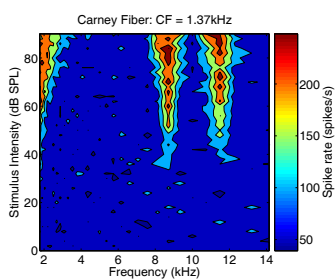

(a)

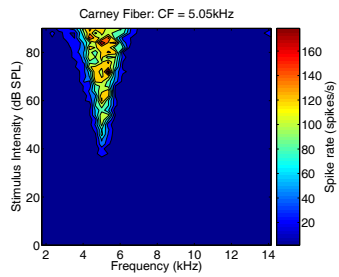

(c)

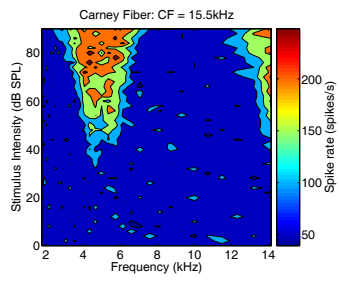

(e)

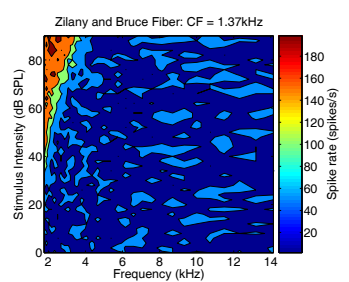

(b)

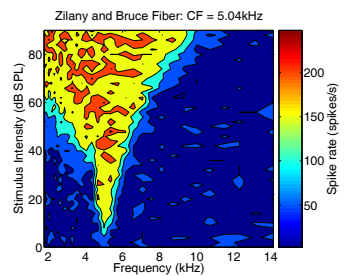

(d)

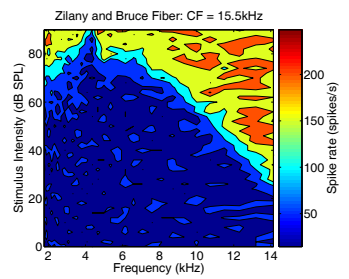

(f)
Fig. 4. Response maps of AN fibers. Carney AN model are shown in subfigures (a), (c) and (e), respectively. Zilany \& Bruce AN model are shown in subfigures (b), (d) and (f), respectively.

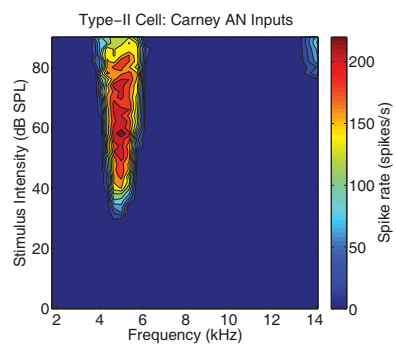

(a)

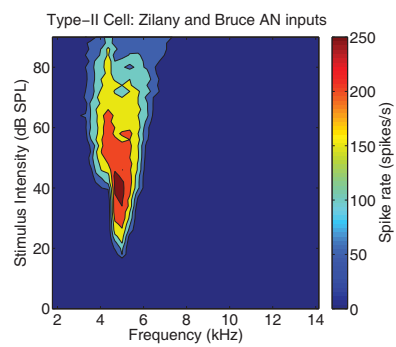

(b)
Fig. 5. Type-II cell responses with identical DCN model parameters. The type-II cells simulated using the Carney and Zilany \& Bruce AN model inputs can be seen in (a) and (b), respectively.

$12-14-\mathrm{kHz}$ range. This region of excitation is well beyond the W-cell input AN fiber bandwidth of 2.0 octaves. This region likely stems from the high frequency distortions in the Carney low-CF fibers shown in Fig. 4. The second thing we notice is that the Zilany \& Bruce W-cell has a slightly wider response with lower excitation thresholds than the Carney $\mathrm{W}$-cell. This is the result of the $4-\mathrm{kHz}$ notch in the middleear filter of the Zilany \& Bruce model. All AN fibers have their responses to frequencies around $4 \mathrm{kHz}$ significantly attenuated and as such cause a decrease in excitation. Away from $4 \mathrm{kHz}$, however, we see that the Zilany \& Bruce W-cells have higher levels of excitation than the Carney W-cells.

The results shown in Figs. 5 and 6 provide us with concrete goals for integrating the two models. We need to increase the levels of excitation at higher stimulus intensities for our type-II cells and narrow their responses. Doing so will allow 


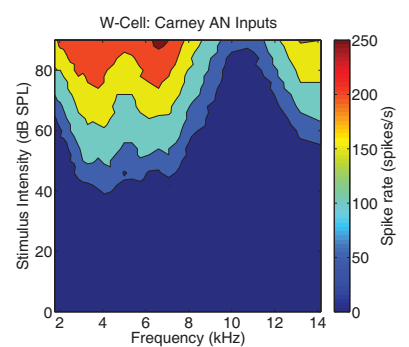

(a)

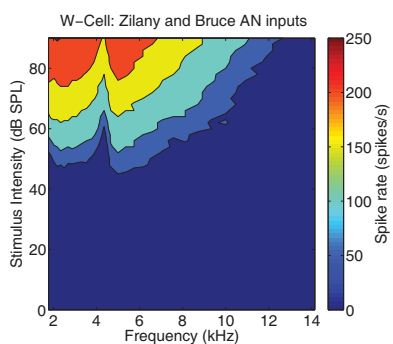

(b)
Fig. 6. W-cell responses with identical DCN model parameters. The Wcells simulated using the Carney and Zilany \& Bruce AN model inputs can be seen in (a) and (b), respectively.

for increased inhibition of P-cells at higher intensities which would help to decrease the regions of excitation and begin to match the two outputs.

Figure 7 shows the effects of certain parameter changes on the responses of type-II cells. In an attempt to increase excitation in type-II cells we first explored decreasing the level of inhibition from input $\mathrm{W}$-cells. The AN connection strength to the W-cells was decreased to 0.04 from 0.06 and the AN connection strength to the type-II cell was decreased to 0.45 from 0.55 in 7 (a). These changes had the desired effects of slightly increased excitability at higher intensities and decreases in the excitation thresholds of the type-II cell.

In Fig. 7(b) we decreased the bandwidth of AN fiber inputs to the type-II cell to 0.3 from 0.4 . This had the effect of increasing the overall excitation but widening the response of the type-II cell. This is likely the result of having greater overlap between the responses of $\mathrm{AN}$ fibers as they begin to get closer and closer.

Finally in Fig. 7(c) we increased the AN fiber connection strength to the type-II cell back to 0.55 and decreased the $\mathrm{W}$-cell connection strength to 1.3. Both of these parameters were changed individually and produced the same result, a widening of the response and increased levels of excitation. Figure 7(c) shows the effect of changing them both together which greatly enhances the behavior.

\section{CONCLUSIONS AND FUTURE WORKS}

\section{A. Conclusions}

The preliminary results in this study show that the change in AN fiber model produces significantly different DCN model responses. Using AN fibers from the Zilany \& Bruce model result in much larger and broader excitatory regions and decreases the size of inhibitory regions in the resulting response maps of DCN cells.

The lower thresholds and broadening tuning at higher SPLs in the AN fibers simulated using the Zilany \& Bruce model cause type-II cell responses that are wider and less excitatory at higher frequencies. Type-II cells are significant inhibitors to all P-cells. Their decreased excitation at higher stimulus levels causes a decrease in the total inhibition of $\mathrm{P}$-cells in those same regions which in turn allows for the increased excitation we see in our simulated P-cells.

The broadening of the excitatory regions can be mitigated by thinning the region of excitation of type-II cells and

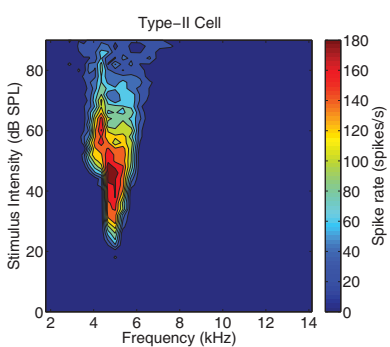

(a)

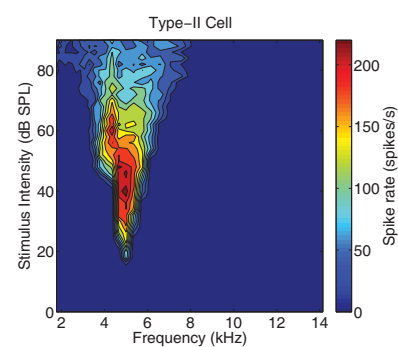

(b)

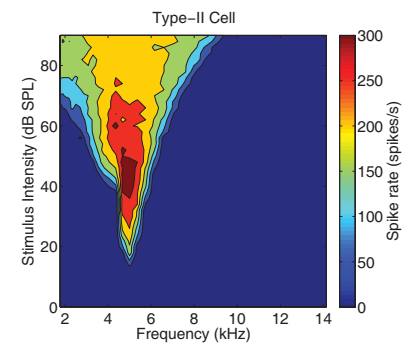

(c)

Fig. 7. Changes to type-II cell responses resulting from changes to DCN model connection parameters. (a) shows the effect of decreasing the AN connection strength to W-cells to 0.04 the type-II response. (b) shows the further effect of decreasing the input AN bandwidth to type-II cells to 0.3 octaves. (c), shows the effects of increasing the AN fiber connection strength to the type-II cell to 0.55 and decreasing the W-cell connection strength to 1.3. The changes from each figure are carried into the next. Zilany and Bruce AN fibers were used as input.

increasing their excitation at higher stimulus intensities. To do so we must find a balance between using changes in AN connection parameters to increase the overall excitability and increases in $\mathrm{W}$-cell inhibition to narrow the response.

\section{B. Future Works}

Using the insights gained in this preliminary study, we aim to find a set of DCN model parameters that will produce physiologically-realistic responses to acoustic stimuli. Once the Zilany \& Bruce AN model and the Zheng \& Voigt DCN model have been fully integrated, we can begin to examine the effects of hearing loss in the auditory periphery on the response properties of DCN cells and gain further insight into the effects of hearing loss on the central auditory system.

\section{REFERENCES}

[1] L. H. Carney, "A model for the responses of low-frequency auditorynerve fibers in cat," The Journal of the Acoustical Society of America, no. 93, pp. 401-417, 1993.

[2] M. S. A. Zilany and I. C. Bruce, "Modeling auditory-nerve responses for high sound pressure levels in the normal and impaired auditory periphery," J. Acoust. Soc. Am., vol. 120, no. 3, pp. 1446-1466, Sept. 2006.

[3] _ , "Representation of the vowel $/ \varepsilon /$ in normal and impaired auditory nerve fibers: Model predictions of responses in cats," J. Acoust. Soc. Am., vol. 122, no. 1, pp. 402-417, July 2007.

[4] X. Zheng and H. F. Voigt, "Computational model of response maps in the dorsal cochlear nucleus," Biol. Cybern., no. 95, pp. 233-242, 2006.

[5] R. J. MacGregor, Neural and brain modeling. San Diego: Academic Press, 1987.

[6] K. A. Davis and H. F. Voigt, "Computer simulation of shared input among projection neurons in the dorsal cochlear nucleus," Biol. Cybern. no. 74, pp. 413-425, 1996.

[7] K. A. Davis, G. T. Gdowski, and H. F. Voigt, "A statistically based method to generate response maps objectively," J. Neurosci., no. 57, pp. 107-118, 1995. 